\title{
Ocorrência de alterações do Processamento Auditivo em crianças com Transtorno de Aprendizagem
}

\author{
Occurrence of Auditory Processing Changes in Children with Learning Disorder
}

\author{
Ocurrencia de alteraciones del Procesamiento Auditivo en niños con Trastorno de \\ Aprendizaje
}

Mariane de Souza Zampieri ${ }^{1 *}$, Jessica Ramos Tavares², Gisele Vieira Hennemann Koury ${ }^{2}$, Juliana Faleiros Paolucci Bigarelli², Dyana Barbosa Ferreira², Isabel Cristina Neves de Souza ${ }^{2,3}$.

\section{RESUMO}

Objetivo: Avaliar a ocorrência de alterações do Processamento Auditivo Central em crianças com Transtorno de Aprendizagem. Métodos: Estudo descritivo, transversal, retrospectivo, realizado no ambulatório de Foniatria do setor de Otorrinolaringologia do Hospital Universitário Bettina Ferro de Souza da Universidade Federal do Pará com 98 crianças escolarizadas de 5 a 12 anos com Transtorno de Aprendizagem, sem alterações neurológicas, cognitivas ou visuais e com exames audiométricos normais, que foram submetidas a testes comportamentais de Processamento Auditivo: Fala com Ruído Branco, Dicótico Não-Verbal, Reconhecimento de Sentenças com Mensagem Competitiva, Dicótico de Dígitos, Dicótico de Dissílabos Alternados (SSW - Staggered Spondaic Words para o português) e RGDT (Random Gap Detection Test). Resultados: $69,4 \%$ eram do sexo masculino e a faixa etária mais acometida foi a de $11-12$ anos (39,8\%). Todas as crianças tiveram pelo menos um dos testes de Processamento Auditivo alterado. Obtiveram resultados alterados significativamente nos testes: Dicótico Não Verbal $(60,2 \%$ na atenção livre; $56,1 \%$ na atenção à direita e 58,2\% na atenção à esquerda), Dicótico de Dígitos (62,2\% em orelha direita e 75,5\% em orelha esquerda), RGDT (68,4\%), SSW quantitativo (63,3\% em orelha direita e $64,3 \%$ em orelha esquerda) e qualitativo (efeito de ordem Baixo/Alto em 27,6\%). Os resultados foram predominantemente normais nos testes: Fala com ruído (70,4\%), Reconhecimento de Sentenças com Mensagem Competitiva (relação sinal/ruído 0db: $65,5 \%$ em orelha direita e $84,5 \%$ em orelha esquerda). Conclusão: Crianças com Transtorno de Aprendizagem apresentaram alterações no Processamento Auditivo, devendo ser mapeadas nestas habilidades para melhor compreensão e direcionamento de uma reabilitação individualizada.

Palavras-chave: Percepção auditiva, Transtornos de aprendizagem, Criança.

\begin{abstract}
Objective: Evaluate the occurrence of changes in Central Auditory Processing in children with Learning Disorder.Methods: Descriptive, cross-sectional, retrospective study was perfomerd at the Phoniatrics medical outpatient of the Otorhinolaryngology Sector of Bettina Ferro de Souza University Hospital of the Federal University of Pará with 98 children enrolled from 5 to 12 years with Learning Disorders, without neurological, cognitive or visual alterations and with normal audiometric tests, who were submitted to behavioral tests of auditory processing: Speech with White Noise, Nonverbal Dichotic, Sentence Recognition in Competitive Message, Dichotic Digits, Alternate Dissyllable Dichotic (SSW - Staggered Spondaic Words to portuguese) and RGDT (Random Gap Detection Test). Results: $69.4 \%$ were males and the age group most affected was 11-12 years $(39.8 \%)$. All children had at least one of the auditory processing tests altered. Results were
\end{abstract}

\footnotetext{
${ }^{1}$ Residente de Otorrinolaringologia no Hospital Universitário Bettina Ferro de Souza, Universidade Federal do Pará (UFPA), Belém-PA *E-mail: marianezampieri@gmail.com

${ }^{2}$ Hospital Universitário Bettina Ferro de Souza, Universidade Federal do Pará (UFPA), Belém-PA.

${ }^{3}$ Professora Adjunta da Universidade Federal do Pará (UFPA), Belém-PA.
} 
significantly altered in the tests: Nonverbal Dichotic $(60.2 \%$ in free attention, $56.1 \%$ in attention to the right and $58.2 \%$ in attention to the left); Dichotic of Digits (62.2\% in right ear and $75.5 \%$ in the left ear); RGDT (68.4\%); quantitative SSW $(63, \%$ in the right ear and $64.3 \%$ in the left ear) and qualitative SSW (27.6\% order effect low/high). They obtained predominantly normal results in the tests: Speech with white noise (70.4\%); Sentence Recognition in Competitive Message (0db ratio signal-to-noise: $65.5 \%$ in the right ear and $84.5 \%$ in the left ear). Conclusion: Children with Learning Disorder presented alterations in auditory processing and should be mapped to a better understanding and direction of individualized rehabilitation.

Keywords: Auditory perception, Learning disorders, Child.

\section{RESUMEN}

Objetivo: Evaluar la ocurrencia de alteraciones del Procesamiento Auditivo Central en niños con Trastorno de Aprendizaje. Métodos: Estudio descriptivo, transversal, retrospectivo, realizado en el ambulatorio de Foniatría del sector de Otorrinolaringología del Hospital Universitario Bettina Ferro de Souza de la Universidad Federal de Pará con 98 niños 5 a 12 años con trastorno de aprendizaje, sin alteraciones neurológicas, cognitivas o visuales y con exámenes audiométricos normales, que fueron presentadas a pruebas conductuales de procesamiento auditivo: Habla en Ruido, Dicótico No Verbale, Reconocimiento de Sentencias con Mensaje Competitivo, Dicótico de Dígitos, Dicótico Disílabo (SSW - Staggered Spondaic Words para o portugués) y RGDT (Random Gap Detection Test). Resultados: 69,4\% eran del sexo masculino y la franja etaria más acometida fue la de 11-12 años (39,8\%). Todos tuvieron al menos una de las pruebas de procesamiento auditivo alterado. Obtuvieron resultados alterados significativamente las pruebas: Dicótico No Verbale $(60,2 \%$ en la atención libre, $56,1 \%$ en la atención a derecha y 58,2\% en la atención a izquierda), Dicótico de Dígitos (62,2\% en oreja derecha y $75,5 \%$ en oreja izquierda), RGDT (68,4\%), SSW cuantitativo ( $63 \%$ en oreja derecha y $64,3 \%$ en oreja izquierda) y cualitativo (efecto orden Bajo / Alto en $27,6 \%$ ). Se obtuvieron resultados predominantemente normales en las pruebas: Habla en ruido $(70,4 \%)$, Reconocimiento de Sentencias con Mensaje Competitivo (relación señal/ruido 0db: $65,5 \%$ en oreja derecha y $84,5 \%$ en oreja izquierda). Conclusión: Niños con trastorno de aprendizaje presentaron alteraciones en el procesamiento auditivo, debiendo ser mapeadas para una mejor comprensión y dirección de una rehabilitación individualizada.

Palabras-clave: Percepción auditiva, Transtornos del aprendizaje, Niño.

\section{INTRODUÇÃO}

As experiências auditivas têm um papel importante na capacidade de comunicação interpessoal, favorecendo a compreensão das informações, o aprendizado e a socialização (ENGELMANN E e FERREIRA MIDC, 2009; ASHA, 2005). Através da transdução dos sinais acústicos ouvidos em impulsos neurais no sistema auditivo periférico e pela posterior interpretação e integração desses impulsos neurais no sistema auditivo central, conseguimos elaborar uma resposta adequada ao estímulo recebido (BSA, 2007).

De acordo com o consenso da American Speech-Language-Hearing Association (ASHA), o Processamento Auditivo (Central) [PA (C)] se refere à eficiência e eficácia com que o Sistema Nervoso Central (SNC) utiliza as informações auditivas (ASHA, 2005). É o processamento perceptivo da informação auditiva no SNC e a atividade neurobiológica que subjaz este processamento, dando origem aos potenciais auditivos eletrofisiológicos e às mudanças nas respostas comportamentais aos sons. O PA (C) inclui os mecanismos auditivos que fundamentam as seguintes capacidades ou habilidades auditivas: localização sonora e lateralização; discriminação auditiva; reconhecimento de padrões auditivos; aspectos temporais da audição; desempenho auditivo em sinais acústicos competitivos e desempenho auditivo com sinais acústicos degradados (ASHA, 1996; BELLIS TJ, 2003; CHERMAK GD e MUSIEK FE, 1997). Estas habilidades nos permitem compreender os estímulos sonoros no "mundo real", onde sempre ocorre algum tipo de competição sonora (RIBAS A, ROSA MRD, KLAGENBERG $\mathrm{K}$ et al., 2007). 
O Transtorno de Processamento Auditivo (Central) [TPA (C)] refere-se a dificuldades no processamento perceptivo da informação auditiva no SNC afetando o desempenho em uma ou mais das habilidades, ocasionando falhas na compreensão integral da mensagem sonora recebida (ASHA, 2005). No TPA (C) ocorrem queixas relacionadas a dificuldade de escutar em ambientes ruidosos, associados ou não a problemas atencionais e de memória, que podem levar ao insucesso escolar em crianças (ABDOLLAHI FZ et al, 2017; FRIDLIN SL, PEREIRA LD, PEREZ AP, 2014; MOORE DR e HUNTER LL, 2013).

Os Transtornos de Aprendizagem são alterações do neurodesenvolvimento de origem organofuncional que afetam significativamente o desempenho escolar em leitura, escrita e/ou aritmética (AMERICAN PSYCHIATRIC ASSOCIATION, 2014; ROTTA NT, OHLWEILER L, RIESGO RS, 2016). Não se referem a um único distúrbio, mas a uma ampla gama de problemas que podem afetar qualquer área do desempenho acadêmico alterando as possibilidades da criança do aprender independente, apesar de possuir condições intelectuais, escolaridade e nível de desenvolvimento para fazê-lo (SMITH C e STRICK L, 2001; MOOJEN S, 2003).

Devido a informação auditiva ser, em conjunto com a visual, o estímulo predominantemente usado no ambiente acadêmico para a passagem de informações e ocorrer muita competição e degradação sonora na sala de aula, principalmente pelo ruído, avaliar se ocorrem alterações do $P A(C)$ nesta população é fundamental. A detecção precoce da ocorrência do TPA(C) e uma correta orientação e intervenção podem minimizar as dificuldades comunicativas e o desempenho tanto no âmbito escolar como nas relações sociais. Sendo assim, este trabalho tem por objetivo avaliar se ocorrem alterações do Processamento Auditivo Central em crianças com Transtorno de Aprendizagem, mapeando as habilidades auditivas comprometidas, a fim de propiciar uma reabilitação mais eficaz.

\section{MÉTODOS}

Todos os sujeitos da presente pesquisa foram estudados segundo os preceitos da Declaração de Helsinque e do código de Nuremberg, respeitando as Normas de Pesquisas envolvendo Seres Humanos (Res. CNS 466/12) do Conselho Nacional de Saúde após a aprovação do Comitê de Ética em Pesquisa do Instituto de Ciências da Saúde da Universidade Federal do Pará - ICS/ UFPA com o número do parecer 1.566 .255 .

Participaram dessa pesquisa 98 escolares com idade entre 5 e 12 anos. O estudo foi realizado no ambulatório de Foniatria do setor de Otorrinolaringologia do Hospital Universitário Bettina Ferro de Souza da Universidade Federal do Pará, no período de junho de 2016 a agosto de 2018.

Foram incluídas crianças de ambos os sexos, com idade entre 5 e 12 anos, com Transtornos de Aprendizagem, com autorização dos pais/responsáveis, que assinaram o Termo de Consentimento Livre e Esclarecido. Foram excluídas as crianças fora da faixa etária estabelecida, com alterações cognitivas, neurológicas, visuais e audiológicas (com limiares audiométricos acima de $25 \mathrm{~dB}$ e índice percentual de reconhecimento de fala na logoaudiometria abaixo de $88 \%$ para monossílabos) ou cujos pais/responsáveis não aceitaram participar da pesquisa.

Todas as crianças foram submetidas a otoscopia com um otoscópio Heine, modelo Mini 3000 seguidas de avaliação audiológica, constando da audiometria tonal, audiometria vocal, impedanciometria, e testes de avaliação comportamental do PA (C). Estes procedimentos foram realizados em cabine acústica (VIBRASOM) com o audiômetro de dois canais (Clinical Audiometric AL40 - Interacoustics) e o impedanciômetro (GSI 38 Auto Tymp - Interacoustics), calibrado de acordo com as especificações do American National Standards Institute (ANSI 3.1.,1991).

Para a avaliação dos testes comportamentais do PA (C), utilizou-se CD player, da marca Panasonic, acoplado ao audiômetro, com o qual foram aplicados os testes de Pereira e Schochat (2011) de Fala com 
Ruído Branco, teste Dicótico não-verbal, teste de Reconhecimento de Sentenças com Mensagem Competitiva (PSI ou SSI), teste Dicótico de Dígitos, teste Dicótico de Dissílabos Alternados (adaptação do SSWStaggered Spondaic Words para o português) e o teste RGDT (Random Gap Detection Test- Expanded, da Auditec Incorporated). De acordo com o tipo de teste a aplicação foi monótica (duas informações sonoras diferentes escutadas na mesma orelha), dicótica (informação sonora diferente para cada orelha aplicada simultaneamente em ambas as orelhas) ou diótica (mesma informação sonora aplicada às duas orelhas).

O Teste de Fala com Ruído Branco avaliou a capacidade do indivíduo reconhecer sons verbais (palavras) degradados parcialmente pelo ruído branco em escuta monótica, com resposta oral.

O Teste Dicótico Não Verbal avaliou a capacidade de diferenciar dois sons não-verbais diferentes (sons da natureza) em escuta dicótica, com resposta de apontar figuras correspondentes aos sons. Este teste foi realizado com atenção livre (a criança podia apontar o som ouvido na orelha direita ou na esquerda) para avaliar se a escuta era equilibrada entre as orelhas (normal) ou havia predileção pela escuta em uma orelha (alterado). Após, foi solicitado atenção aos sons escutados na orelha direita, seguida da atenção aos sons escutados na orelha esquerda, para avaliar se a criança conseguia manter atenção seletiva de informações auditivas.

O Teste de Reconhecimento de Sentenças com Mensagem Competitiva [Pediatric Speech Inteligibillity (PSI) ou Synthetic Sentence Identification (SSI)] avaliou a capacidade de reconhecer sons verbais (frases apresentadas simultaneamente a uma mensagem competitiva (uma história), em escuta monótica, com respostas de apontar figuras ou frases, respectivamente). Foi considerado sinal ou informação alvo (S) as frases e ruído ou informação distratora $(R)$ a história. Os estímulos foram apresentados com intensidade numa relação $\mathrm{S} / \mathrm{R}$ de $\mathrm{OdB},-10 \mathrm{~dB}$ e $-15 \mathrm{~dB}$.

O Teste Dicótico de Dígitos avaliou a capacidade de reconhecer sons verbais (números) em sequência em escuta dicótica com resposta de repetições orais. Neste teste pede-se para o paciente iniciar a repetição dos números ouvidos pela direita e em seguida falar os números ouvidos a esquerda. Depois, inicia-se a repetição oral dos números ouvidos a esquerda, seguida pelos escutados a direita.

O Teste Dicótico de Dissílabos Alternados (SSW adaptado para o português) avaliou a capacidade de reconhecer sons verbais em sequência (2 palavras compostas em cada orelha que na gravação do teste foram parcialmente sobrepostas) em escuta dicótica, com resposta de repetições orais. É um teste com alto conteúdo linguístico, sendo avaliado de forma quantitativa e qualitativa. Na quantitativa, considera-se normal acertos $\geq 90 \%$, grau leve de 80 a $89 \%$ de acertos, grau moderado de 60 a $79 \%$ de acertos e grau severo $\leq$ $59 \%$ de acertos. Na quantitativa, consideram-se as tendências dos erros. As inversões se referem as trocas na sequência das palavras. O efeito auditivo se relaciona a orelha: errar mais quando começa com a orelha direita (efeito auditivo alto/ baixo) ou quando começa pela orelha esquerda (efeito auditivo baixo-alto). $O$ efeito de ordem se relaciona a errar mais as duas primeiras palavras ouvidas (efeito alto/ baixo) ou as duas últimas (efeito baixo alto).

O RGDT avaliou a capacidade do indivíduo de detectar o menor intervalo de tempo entre dois tons puros em apresentação diótica.

As informações da caracterização amostral foram apuradas em banco de dados elaborado no software Microsoff ${ }^{\circledR}$ Office Exce ${ }^{\circledR} 2010$.

A estatística descritiva (porcentagem de acertos) e analítica (Teste Qui-Quadrado Aderência para tabelas univariadas e Teste Qui-Quadrado Independência para tabelas bivariadas) foram realizadas no software BioEstat ${ }^{\circledR}$ 5.3. Para a tomada de decisão, adotou-se o nível de significância $\alpha=0,05$, sinalizando-se com asterisco $\left(^{*}\right)$ os valores significantes.

REAS/EJCH | Vol. 11 (5) | e405 | DOI: https://doi.org/10.25248/reas.e405.2019 Página 4 de 11 


\section{RESULTADOS}

Das 98 crianças estudadas, $69,4 \%(n=68)$ eram do sexo masculino e $30,6 \%(n=30)$ do sexo feminino. A faixa etária predominante foi de 11 a 12 anos $(39,8 \%, n=39)$, o que foi significativo estatisticamente $(p<0,001)$. As demais faixas etárias não tiveram significância estatística e corresponderam a 5,1\% (5-6 anos, $n=5)$, $25,5 \%$ (7-8 anos, $n=25)$ e 29,6\% (9-10 anos, $n=29)$. Com relação aos testes para avaliação do PA (C), todas as crianças tiveram alteração em pelo menos um dos testes.

No teste de Fala com Ruído, 70,4\% ( $n=69)$ das crianças tiveram o teste normal o que foi significativo estatisticamente $(p<0,001)$. Em 25,5\% ( $n=25)$ ocorreu alteração do exame na orelha direita e $26,5 \%(n=26)$ na orelha esquerda. Houve 4,1\% $(n=4)$ que não conseguiram realizar o teste na orelha direita e $3,1 \%(n=3)$ na orelha esquerda.

No Teste Dicótico Não Verbal, a maioria teve alterações significativas: em 60,2\% ( $n=59)$ na atenção livre; em 56,1\% ( $n=55)$ na atenção à direita e em 58,2\% ( $n=57)$ na atenção à esquerda. Houve 12 crianças $(12,2 \%)$ que não conseguiram realizar este teste na atenção livre e 16 crianças $(16,3 \%)$ na atenção direcionada à direita e à esquerda (Gráfico 1).

Gráfico 1 - Resultados do Teste Dicótico Não Verbal em crianças com Transtorno de Aprendizagem atendidas no setor de Otorrinolaringologia do Hospital Universitário Bettina Ferro de Souza no período de junho de 2016 a agosto de 2018 .

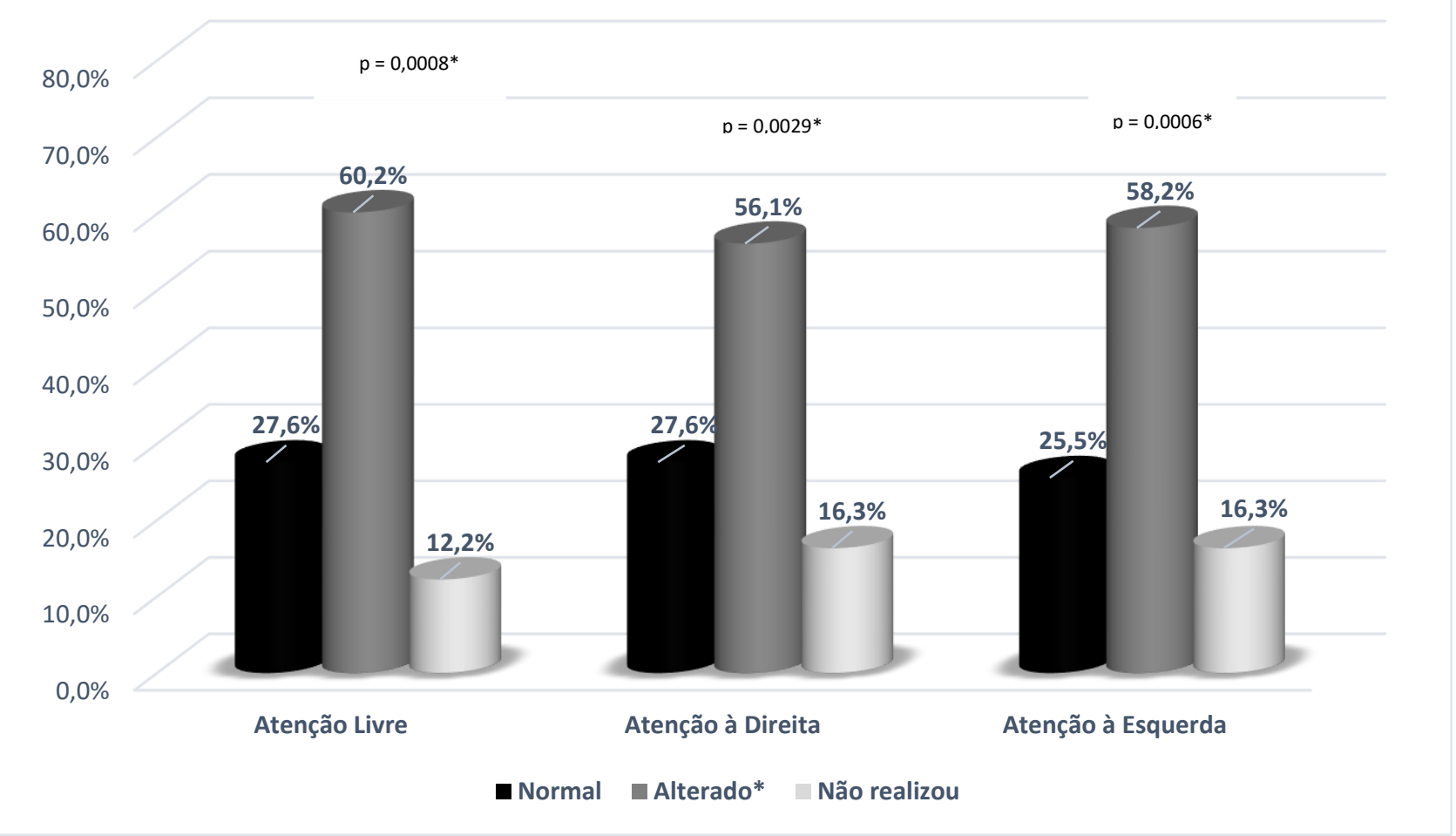

Fonte: Banco de dados da pesquisa.

*Teste Qui-Quadrado Aderência

Para o Teste de Reconhecimento de Sentenças com Mensagem Competitiva, dos 98 pacientes, 86 $(87,8 \%)$ realizaram o PSI, $9(9,2 \%)$ realizaram o SSI e $3(3,1 \%)$ não conseguiram realizar nenhum dos dois testes. Predominaram os resultados normais em ambas as orelhas, não sendo observada diferença significativa estatisticamente nos resultados entre as orelhas (Tabela 1). 
Tabela 1 - Resultados do Teste de Reconhecimento de Sentenças com Mensagem Competitiva em crianças com Transtorno de Aprendizagem atendidos no setor de Otorrinolaringologia do Hospital Universitário Bettina Ferro de Souza no período de junho de 2016 a agosto de 2018

\begin{tabular}{|c|c|c|c|c|c|}
\hline EXAME PSI/SSI & \multicolumn{2}{|c|}{ Orelha Direita (OD) } & \multicolumn{2}{|r|}{ Orelha Esquerda (OE) } & \multirow{2}{*}{$\begin{array}{c}\text { p-valor (OD/OE) } \\
0,5729\end{array}$} \\
\hline S/R: 0 & & & & & \\
\hline Normal* $^{*}$ & 76 & $65,5 \%$ & 71 & $84,5 \%$ & \\
\hline Alterado & 19 & $16,4 \%$ & 23 & $27,4 \%$ & \\
\hline Não realizou & 3 & $2,6 \%$ & 4 & $4,8 \%$ & \\
\hline $\mathbf{p}-$ valor & & $<0.0001^{*}$ & & $<0.0001^{\star}$ & \\
\hline S/R: - 10 & & & & & 0,7713 \\
\hline Normal $^{*}$ & 62 & $53,4 \%$ & 61 & $72,6 \%$ & \\
\hline Alterado & 14 & $12,1 \%$ & 11 & $13,1 \%$ & \\
\hline Não realizou & 22 & $19,0 \%$ & 26 & $31,0 \%$ & \\
\hline $\mathbf{p}-$ valor & & $<0.0001^{\star}$ & & $<0.0001^{*}$ & \\
\hline \multicolumn{6}{|l|}{ S/R: - 15} \\
\hline Normal $^{*}$ & 57 & $49,1 \%$ & 50 & $59,5 \%$ & 0,0981 \\
\hline Alterado & 4 & $3,4 \%$ & 11 & $13,1 \%$ & \\
\hline Não realizou & 37 & $31,9 \%$ & 37 & $44,0 \%$ & \\
\hline p - valor & & $<0.0001^{\star}$ & & $<0.0001^{*}$ & \\
\hline
\end{tabular}

Fonte: banco de dados da pesquisa

${ }^{*} p<0.0001$ Teste Qui-Quadrado Aderência

Gráfico 2 - Resultados do Teste Dicótico de Dígitos em crianças com Transtorno de Aprendizagem atendidos no setor de Otorrinolaringologia do Hospital Universitário Bettina Ferro de Souza no período de junho de 2016 a agosto de 2018.

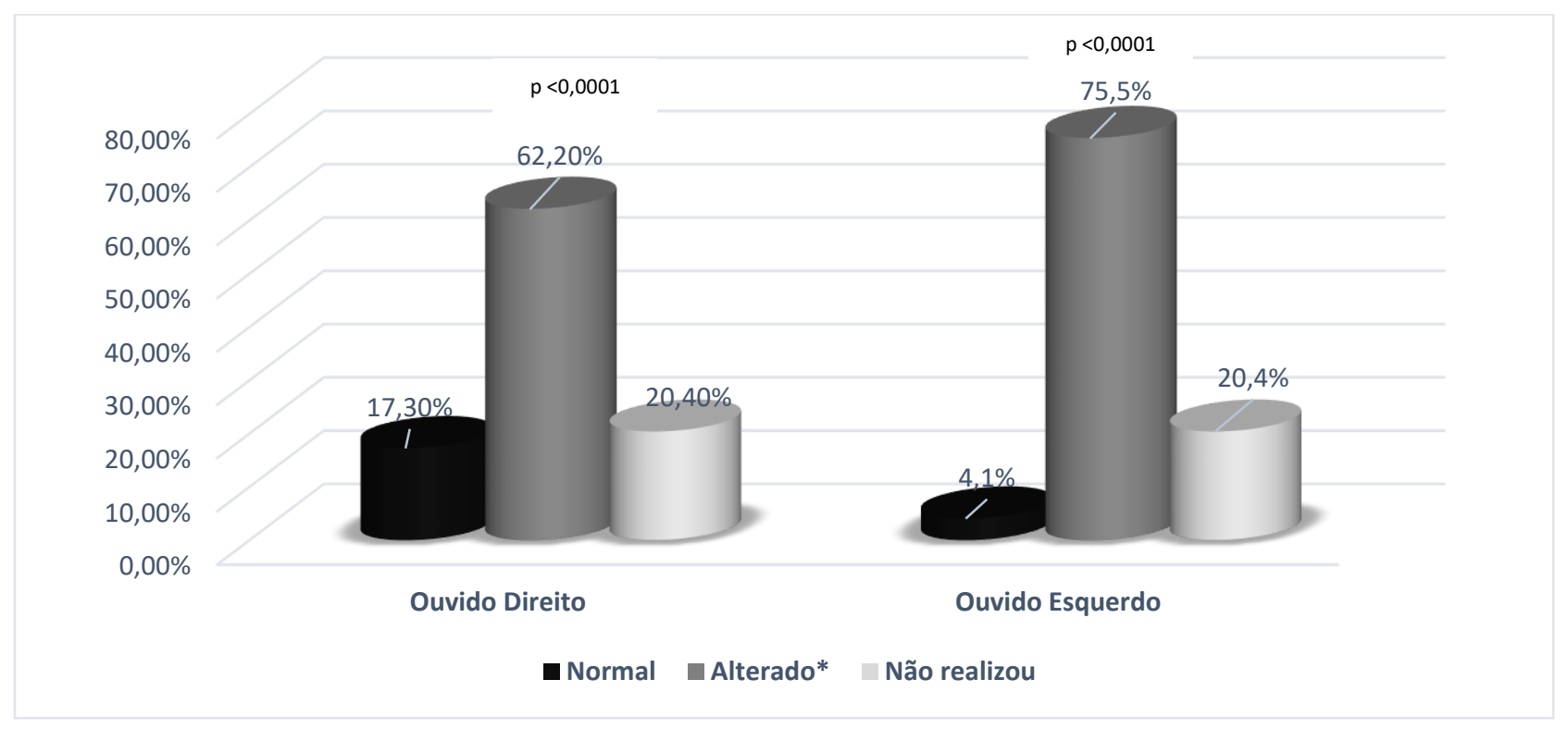

Fonte: Banco de dados da pesquisa

*Teste Qui-Quadrado Aderência p<0,0001.

Gráfico 3 - Resultados do Random GAP Detection Test (RGDT) em crianças com Transtorno de Aprendizagem atendidos no setor de Otorrinolaringologia do Hospital Universitário Bettina Ferro de Souza no período de junho de 2016 a agosto de 2018. 


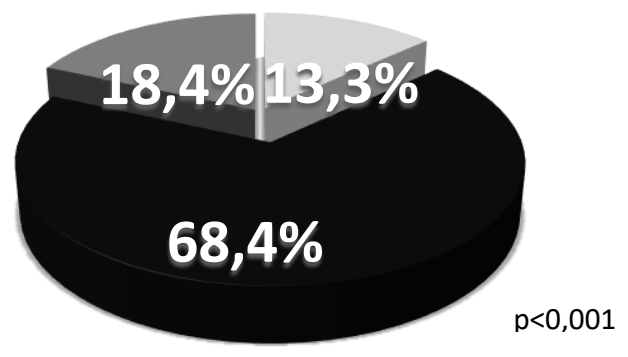

$\square$ Normal $\square$ Alterado* $\square$ Não realizou

Fonte: Banco de dados da pesquisa

*Teste Qui-Quadrado Aderência p<0,0001.

Tabela 2 - Resultados do Teste Dicótico de Dissílabos Alternados em crianças com Transtorno de Aprendizagem atendidos no setor de Otorrinolaringologia do Hospital Universitário Bettina Ferro de Souza no período de junho de 2016 a agosto de 2018

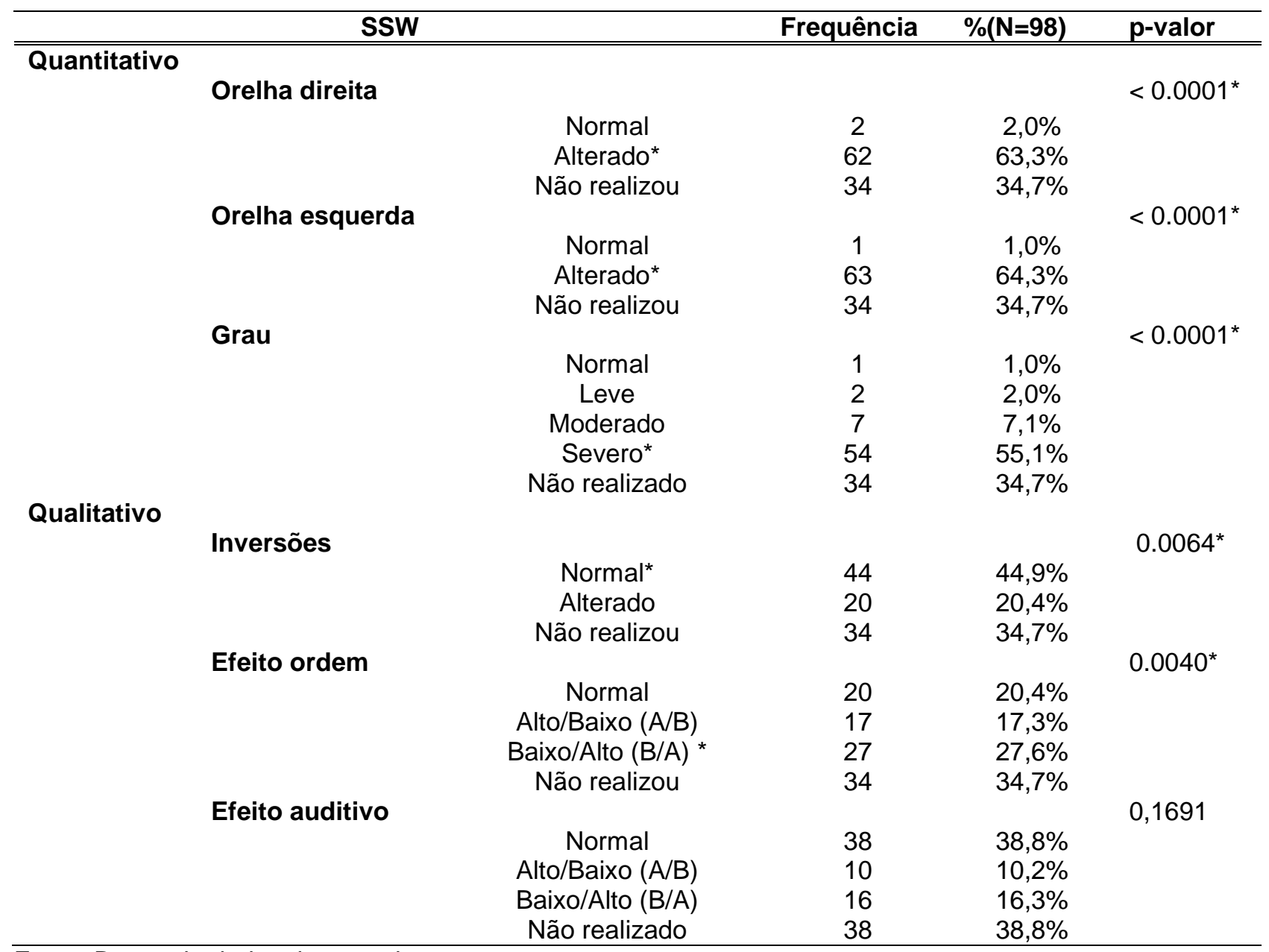

Fonte: Banco de dados da pesquisa

*Teste Qui-Quadrado Aderência significante.

O Teste Dicótico de Dígitos, foi realizado por apenas 78 pacientes (79,6\%), havendo 62,2\% ( $\mathrm{n}=61)$ de falhas em orelha direita e $75,5 \%(n=74)$ em orelha esquerda (Gráfico 2). 
O RGDT foi feito por 80 pacientes, estando $68,4 \%$ ( $n=67)$ com o resultado alterado (Gráfico 3), o que foi significativo estatisticamente $(p<0,0001)$.

Apenas 64 crianças conseguiram fazer o SSW em português. Tiveram o resultado alterado em orelha direita $63,3 \%(n=62)$ e $64,3 \%(n=63)$ em orelha esquerda, sendo que $55,1 \%(n=54)$ tinham grau de alteração severo. Ocorreu número normal de inversões em 44,9\% ( $n=44)$ e em $20,4 \%(n=20)$ houve alteração. Com relação ao efeito ordem, $20,4 \%(n=20)$ tiveram resultado normal e $44,9 \%(n=44)$ alterados $(27,6 \%$ baixo/alto e $17,3 \%$ alto/baixo). Já no efeito auditivo, $38,8 \%(n=38)$ tiveram resultado normal e $26,5 \%(n=26)$ alterado ( $16,3 \%$ baixo/alto e $10,2 \%$ alto/baixo) (Tabela 2$)$.

\section{DISCUSSÃo}

Os resultados dessa pesquisa mostraram uma predominância no gênero masculino (69,4\%), assim como Moojen (2003) e Corona et al. (2005), que também observaram uma maior prevalência de meninos com distúrbio de linguagem e aprendizagem. Também ocorreu uma predominância masculina nas crianças com PA(C) alterado, como observado no estudo de Chermak e Musiek (1992), que estimaram uma predominância masculina de 2:1. Já Dawes et al. (2008), não encontrou diferença significante entre sexo masculino e feminino.

A faixa etária predominante foi de 11 a 12 anos (39,8\%). Moore et al, 2010 mostram que ocorre uma melhora na execução dos testes de $\mathrm{PA}(\mathrm{C})$ com o avançar da idade, ocasionada pela maturação do sistema auditivo. Como esta pesquisa foi realizada na rede pública de saúde, onde existe uma grande dificuldade de acesso a serviços especializados, pode ter ocorrido um diagnóstico em idade mais avançada do que o esperado. Além disso, o aumento da demanda escolar na faixa etária de 11 a 12 anos pode ter aumentado a percepção do Transtorno de Aprendizagem, levando consequentemente a uma procura maior por diagnóstico e tratamento. Se há falhas do desenvolvimento das habilidades do $P A(C)$ e estas não forem detectadas e reabilitadas, ocorrerão progressivas dificuldades no entendimento das informações acadêmicas repassadas principalmente por via oral na sala de aula. Apesar de naturalmente existir desenvolvimento destas funções pela exposição cotidiana às situações sonoras, esta defasagem ocasiona piora no desempenho escolar com o aumento progressivo da demanda acadêmica (SILVA TAG e BARBOSA JSL, 2017). No contexto da educação brasileira atual, a Política de Progressão Escolar Continuada também pode estar colaborando para um encaminhamento mais tardio destas crianças (JACOMINI MA, 2009).

Das crianças estudadas, todas tiveram alteração em pelo menos um dos testes do $P A(C)$. Na literatura também é descrito uma grande prevalência de TPA (C) em crianças com Transtorno do Aprendizagem (Engelmann e Ferreira, 2009). Ribas et al. (2007) pesquisaram o PA(C) de 50 crianças portadoras de Transtornos de Aprendizagem, sendo que $88 \%$ da amostra pesquisada apresentou algum tipo de alteração do $P A(C)$ no exame realizado.

No Teste de Fala com Ruído Branco, $70,4 \%$ das crianças tiveram o teste normal, não sendo observado diferença significativa entre as orelhas. Os resultados encontrados na literatura em relação a isto são conflitantes. Concordando com este estudo, Frota e Pereira (2010), também encontraram uma média de acertos na faixa da normalidade. Já Machado et al. (2011) encontraram predomínio de resultados alterados $(66,67 \%)$ e Neves e Schochat (2005) observaram uma média de acertos abaixo da normalidade em crianças com 8 e 9 anos com dificuldades escolares. A avaliação desta habilidade é importante nos Transtornos de Aprendizagem para avaliar a capacidade de realizar escuta em situações de competição sonora com ruído, que degrada parte das informações sonoras, exigindo do indivíduo a recomposição da mensagem original, o que é denominado fechamento auditivo. Avalia, portanto, o sistema bottom-up de compreensão da fala, ou seja, o caminho da informação auditiva do tronco cerebral em direção ao córtex (O'BEIRNE GA, MCGAFFIN AJ, RICKARDL NA, 2012). 
Quanto ao Teste Dicótico Não Verbal, a maioria teve seu resultado alterado: em 60,2\% na atenção livre; em $56,1 \%$ na atenção à direita; em $58,2 \%$ na atenção à esquerda, não sendo observado diferença estatisticamente significante entre atenção à direita e à esquerda (Gráfico 1), assim como no estudo de Frota e Pereira (2010). Este teste auxilia no entendimento de como a mensagem sonora não verbal é compreendida. Sua falha expõe dificuldades em reconhecer padrões sonoros não verbais, avaliando na atenção livre, se ocorre equilíbrio entre as orelhas na percepção de mensagens auditivas e na atenção direcionada, se há atenção seletiva para as mensagens. Estas habilidades são importantes pois auxiliam na compreensão pragmática da informação auditiva, já que a melodia da fala se altera em afirmações, negações, perguntas e exclamações, podendo ter influência na compreensão de ambiguidades de sentido, de metáforas e interpretação de piadas (FROTA S e PEREIRA LD, 2010).

No Teste de Reconhecimento de Sentenças com Mensagem Competitiva ( $P S I$ e SSI), foi observado predomínio de respostas normais em ambas as orelhas, não havendo diferença estatisticamente significante entre elas (Tabela 1). Já no estudo de Garcia, Pereira e Fukuda (2007) houve diferença estatisticamente significante apenas entre os resultados obtidos à orelha direita e orelha esquerda na relação fala/ruído de $15 \mathrm{~dB}$. No estudo por nós realizado, dos 98 pacientes estudados, 86 realizaram o PSI (com figuras). Destes, 71 estavam na faixa etária onde seria mais adequado realizar o SSI (com sentenças) pois a leitura já devia estar instalada e fluente. Como estes pacientes não tinham a habilidade de leitura necessária, foi realizado o $P S I$, considerado teste mais adequado para ser realizado de 4 a 7 anos segundo o manual de Pereira e Schochat (1997). Isso pode explicar o maior índice de normalidade obtido no resultado do teste, mas mostra um atraso no desenvolvimento das habilidades acadêmicas esperadas.

No Teste Dicótico de Dígitos foi realizado por apenas 78 crianças. Destas, 62,2\% ( $n=61)$ falharam em orelha direita e $75,5 \%$ ( $n=74$ ) falharam em orelha esquerda (Gráfico 2). No estudo de Pinheiro e Capellini (2009), realizado com crianças com e sem Transtorno de Aprendizagem, foi observado um desempenho pior das crianças com transtorno, o que revela uma ineficácia da integração interhemisférica de informações auditivas e dificuldade de reconhecimento de sons verbais em escuta dicótica em tarefa de atenção sustentada (OBRZUT JE e MAHONEY EB, 2011).

No $R G D T$, das 80 crianças que conseguiram realizar o teste, $68,4 \%$ ( $n=67)$ tiveram o resultado alterado (Gráfico 3). Dias et al (2012), encontraram uma taxa de falha no RGDT em $48 \%$ das crianças com TPA(C). Os resultados mostraram que $86 \%$ das crianças com $5-6$ anos, $49 \%$ com $7-8$ anos e cerca de $30 \%$ dos indivíduos com 9 anos tiveram resultados anormais. Algumas dessas crianças podem ter dificuldades no processamento da fala por causa da sua capacidade limitada para perceber pequenos intervalos de silêncio entre os segmentos de fala. $O$ processamento verbal da fala depende, em parte, das habilidades de processamento temporal. Este reconhecimento é importante para detectar entonação, ritmo, duração e diferença de tempo de emissão de fonemas, sendo base dos distúrbios específicos de linguagem que interferem no aprendizado da leitura e escrita (BALEN SA et al., 2009).

Apenas 64 crianças conseguiram fazer o SSW em português (Tabela 2). Tiveram o resultado alterado em orelha direita $63,3 \%(n=62)$ e $64,3 \%(n=63)$ em orelha esquerda. Frota e Pereira (2010) e Schimidt et al. (2007), comparando um grupo de crianças com e sem transtorno de aprendizagem com o teste SSW também observaram um desempenho pior no primeiro grupo, nas tarefas de discriminação de sons sobrepostos em escuta dicótica, o que também foi descrito por Schimidt et al. (2007). Na avaliação quantitativa houve predominância do grau severo (acertos $\leq 59 \%$ ). Grande parte das crianças não conseguiu realizá-lo (34,7\%). A maior carga linguística envolvida no SSW, associada a presença de Transtornos de Aprendizagem pode estar associada a esta ocorrência.

$\mathrm{Na}$ avaliação qualitativa das tendências dos erros ocorreu predomínio significativo de normalidade no quesito inversão, predomínio não significativo de normalidade no efeito auditivo e predomínio significativo de alteração no efeito de ordem. Alterações de inversão se referem às trocas na sequência das palavras, indicando dificuldades para sons verbais em sequência, podendo dificultar o aprendizado da leitura e escrita 
e ocasionar alterações na compreensão do discurso (FROTA S e PEREIRA LD, 2010). O efeito de ordem se relaciona a errar mais as duas primeiras palavras ouvidas (efeito alto/ baixo) ou as duas últimas (efeito baixoalto). O efeito de ordem alto/baixo é indicativo de alterações na memória auditiva, havendo desconforto em situações ruidosas e necessidade de "pistas" para completar informações. O efeito de ordem baixo-alto se relaciona a dificuldades na análise e síntese auditiva, estando envolvidos com alterações na linguagem compreensiva (PEREIRA LD e SCHOCHAT E, 2011).

\section{CONCLUSÃO}

Os Transtornos de Aprendizagem avaliados em nosso estudo predominaram no gênero masculino, na faixa etária de 11-12 anos, apresentando pelo menos um teste alterado na avaliação comportamental do PA(C). Houve predominância significativa de normalidade nos testes monóticos de Fala com Ruído Branco e de Reconhecimento de Sentenças com Mensagem Competitiva ( $P S I$ e SSI), havendo alterações no Teste Dicótico Não Verbal, Dicótico de Dígitos, RGDT e SSW. O mapeamento das habilidades auditivas centrais em pacientes com Transtornos de Aprendizagem pode auxiliar na compreensão e direcionar a reabilitação de forma individualizada.

\section{REFERÊNCIAS}

1. ABDOLLAHI FZ, LOFTI Y, MOOSAVI A et al. Binaural interaction component of middle latency response in children suspected to central auditory processing disorder. Indian Journal of Otolaryngology and Head \& Neck Surgery, 2017; $1-4$.

2. AMERICAN PSYCHIATRIC ASSOCIATION. Manual diagnóstico e estatístico de transtornos mentais. 5 $5^{\mathrm{a}}$ ed. Porto Alegre: Artmed, 2014; 992p.

3. ASHA: American Speech-Language-Hearing Association. Central auditory processing: Current status of research and implications for clinical practice. American Journal of Audiology, 1996; 5(2): 41-54.

4. ASHA: American Speech-Language-Hearing Association. (Central) Auditory Processing Disorders [Technical Report], 2005; 20p.

5. AYRES M, AYRES JÚNIOR M, AYRES DL et al. BioEstat: aplicações estatísticas nas áreas das ciências biológicas e médicas. Belém; Sociedade Civil Mamirauá: MCT-CNPq, 2010; 364p.

6. BALEN SA, BRETZKE L, MOTTECY, CM et al. Resolução temporal de crianças: comparação entre audição normal, perda auditiva condutiva e distúrbio do processamento auditivo. Revista Brasileira de Otorrinolaringologia, 2009; 75(1): 123-129.

7. BELLIS TJ. Assessment and management of central auditory processing disorders in the educational setting: From science to practice. 2nd ed. San Diego: Singular, 2003; 552p.

8. BSA: British Society of Audiology. Auditory Processing Disorder (APD), 2007

9. CHERMAK GD, MUSIEK FE. Managing central auditory processing disorders in children and youth. American Journal of Audiology, 1992; 1(3): 61-66.

10. CHERMAK GD, MUSIEK FE. Central auditory processing disorders: New perspectives. San Diego: Singular, 1997; 374p.

11. CORONA AP, PEREIRA LD, FERRITE S et al. Memória sequencial verbal de três e quatro sílabas em escolares. Pró-Fono, 2005;17(1):27-36.

12. DAWES $P, B I S H O P$ DVM, SIRIMANNA $T$ et al. Profile and aetiology of children diagnosed with Auditory Processin Disorder (APD). International Journal of Pediatric Otorhinolaryngology, 2008; 72(4): 483-489.

13. DIAS KZ, JUTRAS B, ACRANI IO et al.. Random Gap Detection Test (RGDT) performance of individuals with central auditory processing disorders from 5 to 25 years of age. International Journal of Pediatric Otorhinolaryngology, 2012;76(2): 174-178.

14. ENGELMANN E, FERREIRA MIDC. Avaliação do processamento auditivo em crianças com dificuldades de aprendizagem. Revista da Sociedade Brasileira de Fonoaudiologia, 2009; 14(1): 69-74.

15. FRIDLIN SL, PEREIRA LD, PEREZ AP. Relação entre dados coletados na anamnese e distúrbio do processamento auditivo. Revista CEFAC, 2014; 16(2): 405-412.

16. FROTA S, PEREIRA LD. Processamento auditivo: estudo em crianças com distúrbios da leitura e da escrita. Revista Psicopedagogia, 2010; 27(83): 214-222.

17. GARCIA VL, PEREIRA LD, FUKUDA Y. Atenção seletiva: PSI em crianças com distúrbio de aprendizagem. Revista Brasileira de Otorrinolaringologia, 2007; 73(3):404-411. 
18. JACOMINI MA. Educar sem reprovar: desafio de uma escola para todos. Educação e Pesquisa, São Paulo, 2009; 35 (3): 557-572.

19. MACHADO CSS, VALLE HLBS, PAULA KM et al. Caracterização do processamento auditivo das crianças com distúrbio de leitura e escrita de 8 a 12 anos em tratamento no centro clínico de fonoaudiologia da pontifícia universidade católica de minas gerais. Revista CEFAC, 2011; 13(3): 504-512.

20. MOOJEN S. Caracterizando os transtornos de aprendizagem. In: BASSOLS AMS, SANTIS MFB, SUKIENNIK PB et al. Saúde mental na escola 1: uma abordagem multidisciplinar. Porto Alegre: Mediação, 2003; 98-110.

21. MOORE DR, FERGUSON MA, EDMONDSON-JONES AM et al. Nature of Auditory Processing Disorder in Children. Pediatrics, 2010; 126(2): e382 - e390

22. MOORE DR, HUNTER LL. Auditory processing disorder (APD) in children: a marker of neurodevelopmental syndrome. Hearing, Balance and Communication, 2013; 11: 160-167.

23. MORAIS J. O leitor fracassado. In: MORAIS J. A arte de ler. São Paulo: Ed. da Universidade Estadual Paulista, 1996; 211-216.

24. NEVES IF, SCHOCHAT E. Maturação do processamento auditivo em crianças com e sem dificuldades escolares. Pró-Fono, 2005; 17(3): 311-320.

25. O'BEIRNE GA, MCGAFFIN AJ, RICKARDL NA. Development of an adaptive low-pass filtered speech test for the identification of auditory processing disorders. International Journal of Pediatric Otorhinolaryngology, $2012 ; 76$ (6): 777-782.

26. OBRZUT JE, MAHONEY EB. Use of the dichotic listening technique with learning disabilities. Brain and Cognition, 2011; 76(2): 323-331.

27. PEREIRA LD, SCHOCHAT E. Processamento auditivo central: manual de avaliação. São Paulo: Lovise; 1997: 99-138.

28. PEREIRA LD, SCHOCHAT E. Testes Auditivos Comportamentais para Avaliação do Processamento Auditivo Central. São Paulo: Pró-Fono, 2011; 82 p.

29. PINHEIRO FH, CAPELLINI SA. Desenvolvimento das habilidades auditivas de escolares com distúrbio de aprendizagem, antes e após treinamento auditivo, e suas implicações educacionais. Revista Psicopedagogia, 2009; 26(80): 231-241.

30. RIBAS A, ROSA MRD, KLAGENBERG K. Avaliação do processamento auditivo em crianças com dificuldades de aprendizagem. Revista psicopedagogia, 2007; 24(73): 2-8.

31. ROTTA NT, OHLWEILER L, RIESGO RS. (Org.). Transtornos da aprendizagem: abordagem neurobiológica e multidisciplinar. 2.ed. Porto Alegre: Artmed, 2016; 512p.

32. SCHMIDT R, WINTER K, TESCH-ROMER $\mathrm{C}$ et al. Are the auditory processing and perception disorder in children with dyslexia? Laryngorhinootologie, 2007; 86(1): 22-6.

33. SILVA TAG, BARBOSA JSL. Distúrbio do Processamento Auditivo Central: A Importância do Diagnóstico Precoce para o Desenvolvimento da Criança. Encontro Internacional de Formação de Professores e Fórum Permanente de Inovação Educacional, 2017; 10 (1): 1-16.

34. SMITH C, STRICK L. Dificuldades de aprendizagem de A a Z: um guia completo para pais e educadores. Porto Alegre, RS: Artmed; 2001; 398p. 